\title{
Thermalization of heavy quarks in the quark-gluon plasma
}

\author{
Hendrik van Hees and Ralf Rapp \\ Cyclotron Institute, Texas A\&M University, College Station, Texas 77843-3366
}

(Received 10 December 2004; published 25 March 2005)

\begin{abstract}
Charm- and bottom-quark rescattering in a quark-gluon plasma (QGP) is investigated with the objective of assessing the approach toward thermalization. Employing a Fokker-Planck equation to approximate the collision integral of the Boltzmann equation we augment earlier studies based on perturbative parton cross sections by introducing resonant heavy-light quark interactions. The latter are motivated by recent QCD lattice calculations that indicate the presence of "hadronic" states in the QGP. We model these states by colorless (pseudo-) scalar and (axial-) vector $D$ and $B$ mesons within a heavy-quark effective theory framework. We find that the presence of these resonances at moderate QGP temperatures substantially accelerates the kinetic equilibration of $c$ quarks as compared to using perturbative interactions. We also comment on consequences for $D$-meson observables in ultrarelativistic heavy-ion collisions.
\end{abstract}

DOI: 10.1103/PhysRevC.71.034907

PACS number(s): 12.38.Mh, 24.85.+p, 25.75.Nq

\section{INTRODUCTION}

Hadrons containing heavy quarks are valuable probes of the strongly interacting matter produced in high-energy collisions of heavy nuclei. The spectral properties of (bound) $c-\bar{c}$ states, such as their binding energy and (decay) width, are expected to undergo substantial modifications in a quark-gluon plasma (QGP) and thus affect charmonium yields and momentum spectra in heavy-ion reactions with QGP formation (see, e.g., Refs. [1-3] for overviews). Reinteractions of individual $c$ quarks in the QGP will reflect themselves in transversemomentum $\left(p_{T^{-}}\right)$spectra of open charm hadrons ( $D$ mesons) [4-7], most notably their elliptic flow, $v_{2}\left(p_{T}\right)$, in semicentral collisions [8,9]. Preliminary experimental results from the BNL Relativistic Heavy-Ion Collider (RHIC) indicate the possibility that the $D$-meson $v_{2}$ could be similar in magnitude to the one of light hadrons $[10,11]$. Since the $c$ quark is rather heavy, this would be quite remarkable and could provide important insight into (nonperturbative) properties of the QGP at moderate temperatures, $T \simeq 1-2 T_{c}$. For example, in the light-quark sector, parton rescattering through hadron-like states in the QGP (motivated by recent lattice calculations of QCD at finite temperature) has been suggested as a mechanism to enhance partonic cross sections [12-14] to facilitate rapid thermalization of the bulk matter at RHIC as required in hydrodynamical models. The notion of charmonium resonances in the QGP $[15,16]$ has been applied earlier to assess $J / \psi$ production at the CERN super proton synchrotron (SPS) and RHIC [17]. Employing kinetic rate equations to consistently account for both dissociation and regeneration reactions, $c+\bar{c} \leftrightarrow J / \psi+X, J / \psi$ resonance formation in the QGP via $c-\bar{c}$ "coalescence" turns out to be the dominant contribution to the final yield in central Au-Au collisions at RHIC [17-19] (see also Refs. [20,21]). Furthermore, $J / \psi$ production was found to be sensitive to the in-medium properties of charm quarks, that is, their in-medium masses and degree of kinetic equilibration $[17,22,23]$.

Early studies of charm-quark thermalization in the QGP have been conducted in Ref. [24] based on elastic perturbative QCD (pQCD) cross sections [25], $c+q(\bar{q}, g) \rightarrow c+q(\bar{q}, g)$, implemented into a Fokker-Planck equation to approximate the collision integral of the Boltzmann equation (see Ref. [26] for a recent application to light partons). With a strong coupling constant $\alpha_{s}=0.6$, rather short relaxation times of around $\sim 4 \mathrm{fm} / c$ have been found for a massless QGP at temperatures $T \simeq 400 \mathrm{MeV}$. However, since the relaxation times are essentially proportional to $\alpha_{s}^{-2}$, more moderate values of $\alpha_{s}$ (e.g., 0.3) lead to a significant increase (by a factor of 3-4), rendering thermalization of $c$ quarks under RHIC conditions unlikely [27]. This is also consistent with recent transport studies [28,29].

In the present article we evaluate heavy-quark rescattering in the QGP via " $D$ "- and " $B$ "-meson resonances, the existence of which is the main assumption in our work. Although lattice QCD has not yet addressed in-medium heavy-light $(Q-\bar{q})$ spectral functions at finite temperature, resonance-like correlations in the QGP are quite plausible in view of the indications for both $q-\bar{q}[30,31]$ and $Q-\bar{Q}$ systems. Further support for this assumption is provided by calculations using effective four-quark interactions within Nambu-Jona-Lasinio models [32-34]. With form factors and coupling constants adjusted to free $D$-meson masses, finitetemperature calculations lead to resonances above the $Q-\bar{q}$ threshold in the QGP, with appreciable widths of several hundred $\mathrm{MeV}$.

Our article is organized as follows: In Sec. II we introduce. Lagrangians for resonant $c-q$ interactions based on chiral and heavy-quark symmetry for scalar, pseudoscalar, vector, and axialvector multiplets (Sec. II A) and evaluate pertinent scattering amplitudes (Sec. II B). In Sec. III we apply the resulting cross sections within a Fokker-Planck equation; we first determine temperature and momentum dependencies of drag and diffusion constants in a static QGP (Sec. III A), and then evaluate the time evolution of $c$-quark transverse-momentum $\left(p_{T}\right)$ spectra within an expanding fireball model to simulate conditions in heavy-ion collisions at RHIC (Sec. III B). We conclude in Sec. IV, which includes a discussion on opencharm observables in ultrarelativistic heavy-ion collisions (URHICs). 


\section{D MESONS IN THE QUARK-GLUON PLASMA}

\section{A. Heavy-light quark Lagrangians}

Our description of $D$-meson resonances in the QGP is based on a rather simplistic quark-meson model, accounting, however, for the relevant symmetries, that is, chiral symmetry in the light quark sector $(u-d)$ and heavy-quark symmetry for $c$ quarks. As a minimal set of resonances, consistent with lattice calculations [30], we assume the lowest lying pseudoscalar $(D)$ and vector mesons $\left(D^{*}\right)$ to survive above $T_{c} .{ }^{1}$ In addition, (approximate) restoration of chiral symmetry mandates the existence of pertinent $\mathrm{SU}(2)_{f}$ chiral partners in the scalar $\left(D_{0}^{*}\right)$ and axial-vector $\left(D_{1}\right)$ channel with mass and width identical to $D$ and $D^{*}$, respectively. The effective Lagrangian thus takes the form

$$
\begin{aligned}
& \mathscr{L}_{D c q} \\
& =\mathscr{L}_{D}^{0}+\mathscr{L}_{c, q}^{0}-i G_{S}\left(\bar{q} \Phi_{0}^{*} \frac{1+\psi}{2} c-\bar{q} \gamma^{5} \Phi \frac{1+\not}{2} c+\text { h.c. }\right) \\
& \quad-G_{V}\left(\bar{q} \gamma^{\mu} \Phi_{\mu}^{*} \frac{1+\not}{2} c-\bar{q} \gamma^{5} \gamma^{\mu} \Phi_{1 \mu} \frac{1+\not}{2} c+\text { h.c. }\right),
\end{aligned}
$$

where h.c. designates the hermitean conjugate. The free (kinetic and mass) terms for quarks and $D$ mesons read

$$
\begin{aligned}
\mathscr{L}_{c, q}^{0}= & \bar{c}\left(\mathrm{i} \not \partial-m_{c}\right) c+\bar{q} \mathrm{i} \not \partial q, \\
\mathscr{L}_{D}^{0}= & \left(\partial_{\mu} \Phi^{\dagger}\right)\left(\partial^{\mu} \Phi\right)+\left(\partial_{\mu} \Phi_{0}{ }^{* \dagger}\right)\left(\partial^{\mu} \Phi_{0}^{*}\right) \\
& -m_{S}^{2}\left(\Phi^{\dagger} \Phi+\Phi_{0}^{* \dagger} \Phi_{0}^{*}\right)-\frac{1}{2}\left(\Phi_{\mu \nu}^{* \dagger} \Phi^{* \mu \nu}\right. \\
& \left.+\Phi_{1 \mu \nu}^{\dagger} \Phi_{1}^{\mu \nu}\right)+m_{V}^{2}\left(\Phi_{\mu}^{* \dagger} \Phi^{* \mu}+\Phi_{1 \mu}^{\dagger} \Phi_{1}^{\mu}\right) .
\end{aligned}
$$

The fields $\Phi$ represent anti-D mesons, transforming as isospinors under isospin rotations.

The interaction terms in Eq. (1) will be evaluated to leading order in $1 / m_{c}$ according to heavy-quark effective theory (HQET) [35]. This ensures the absence of unphysical (four-dimensional longitudinal) degrees of freedom for massive (axial-) vector meson fields as encoded in the transversality constraints

$$
v_{\mu} \Phi^{* \mu}=v_{\mu} \Phi_{1}^{\mu}=0
$$

where $v_{\mu}$ is the four-velocity of the charm quark or $D$ meson. Equivalent relations hold for the $D$-meson self-energies (to leading order in the $1 / m_{c}$ expansion of HQET).

In the light-flavor sector the relevant symmetry is the invariance of the Lagrangian under the chiral $\mathrm{SU}(2)_{L} \times$ $\mathrm{SU}(2)_{R}$ group. For infinitesimal angles $\delta \vec{\phi}_{V, A}$, it is characterized by the standard vector and axial-vector transformations acting on the quark fields as

$$
q \rightarrow\left(1+i \delta \vec{\phi}_{V} \vec{t}+i \delta \vec{\phi}_{A} \vec{t} \gamma_{5}\right) q, \quad c \rightarrow c,
$$

with $\vec{t}=\vec{\tau} / 2$ the generators of SU(2), where $\vec{\tau}$ are the Pauli matrices. To construct the group operations for the $\bar{D}$ fields,

\footnotetext{
${ }^{1}$ In vacuum these are associated with $D^{+}(1870), D^{0}(1865)$, and $D^{*}(2010)$ mesons.
}

we identify the latter with underlying quark currents according to

$$
\Phi \sim \bar{c} \gamma_{5} q, \quad \Phi_{0}^{*} \sim \bar{c} q, \quad \Phi_{\mu}^{*} \sim \bar{c} \gamma_{\mu} q, \quad \Phi_{1, \mu} \sim \bar{c} \gamma_{\mu} \gamma_{5} q .
$$

Here, " " denotes "transforms under (4) like." Thus the transformation rules for the $\bar{D}$-meson fields follow as

$$
\begin{gathered}
\Phi \rightarrow \Phi+i \delta \vec{\phi}_{A} \cdot \vec{t} \Phi+i \delta \vec{\phi}_{V} \cdot \vec{t} \Phi_{0}^{*}, \\
\Phi_{0}^{*} \rightarrow \Phi_{0}^{*}+i \delta \vec{\phi}_{A} \cdot \vec{t} \Phi_{0}^{*}+i \delta \vec{\phi}_{V} \cdot \vec{t} \Phi .
\end{gathered}
$$

With these properties the Lagrangian, Eq. (1), is a scalar under chiral transformations.

For $D_{s}$ mesons we restrict ourselves to the (experimentally known) pseudoscalar and vector states since spontaneous chiral symmetry breaking in the strange-quark sector is expected to persist to temperatures significantly larger than $T_{c}$ (characterized by sizable values of the strange condensate $\langle\bar{s} s\rangle)$. The corresponding Lagrangian is therefore taken to be

$$
\begin{aligned}
\mathscr{L}_{s}= & \left(\partial_{\mu} \Phi_{s}^{\dagger}\right)\left(\partial^{\mu} \Phi_{s}\right)-m_{D_{s}}^{2} \Phi_{s}^{\dagger} \Phi_{s}-\frac{1}{2} \Phi_{s \mu \nu}^{* \dagger} \Phi_{s}^{\mu \nu} \\
& +m_{D_{s}^{*}}^{2} \Phi_{s \mu}^{* \dagger} \Phi_{s}^{\mu}-i G_{s, S} \bar{s} \gamma^{5} \Phi_{s} \frac{1+\psi}{2} c \\
& -G_{s, V} \bar{s} \gamma^{\mu} \Phi_{s, \mu}^{*} \frac{1+\not}{2} c .
\end{aligned}
$$

Equations (1) and (7) constitute our basic vertices for effective charm-light quark interactions in the QGP. (Analogous expressions hold in the $b$-quark sector upon the replacement $c \rightarrow b$ and $D \rightarrow B$.) The underlying parameters, that is, the bare masses of the $D$ meson and their coupling strength to the quarks, will be fixed to resemble more microscopic model calculations of corresponding spectral functions above $T_{c}$, as discussed later. In the following we will also impose the spin symmetry of HQET, implying that the spectral properties of pseudoscalar and vector mesons, and consequently their masses and couplings to quarks, are equal.

\section{B. Meson self-energies and heavy-light quark scattering amplitudes}

The key ingredient for the heavy-quark scattering amplitudes are the heavy-meson exchange propagators,

$$
D_{D, B}(k)=\frac{1}{k^{2}-m_{D, B}^{2}-\Pi_{D, B}(k)},
$$

which are essentially determined by the underlying self-energies, $\Pi_{D, B}$, together with the bare resonance masses, $m_{D, B}$, of the Lagrangian. In the following, we will evaluate the self-energies in terms of the heavy-light-quark loop (cf. the left diagram in Fig. 1), thereby investigating two different schemes to regularize the divergent loop integrals to assess the robustness of our results. We will concentrate again on the charm-quark case, but completely analogous expressions apply to the bottom sector.

Within the dimensional regularization scheme (see, e.g., [36]), the interaction vertices of Eq. (1) yield a self-energy for 

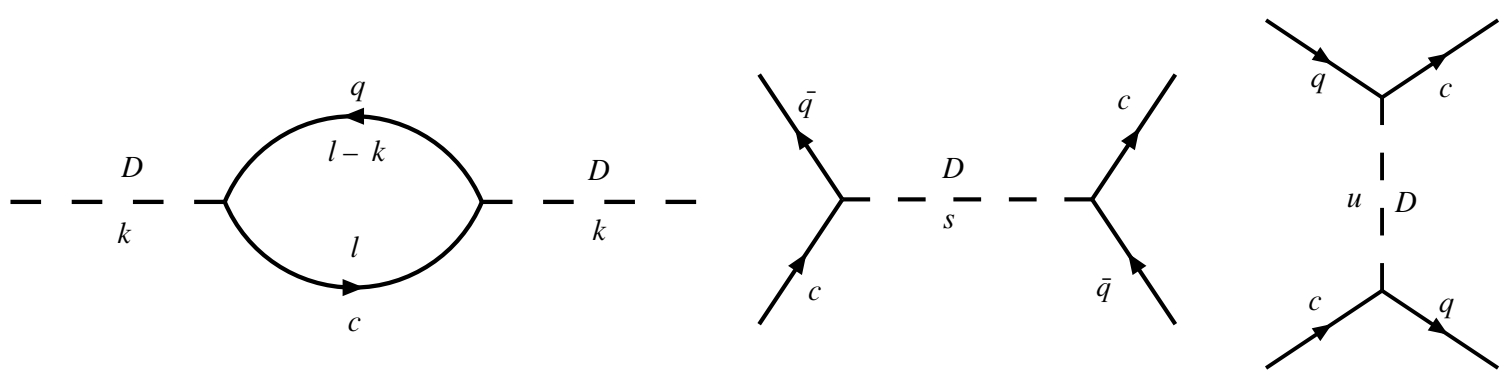

FIG. 1. (Left panel) $c(b)-q$ loop diagram representing the $D(B)$-meson self-energy in the QGP. (Right panel) "Meson"-exchange diagrams contributing to the invariant matrix elements for the scattering of charm quarks on light quarks ( $u$ channel) and antiquarks $(s$ channel).

(pseudo-) scalar $D$ mesons of four-momentum $k$ of the form

$$
\begin{aligned}
\Pi_{D}(s)= & \Pi_{D_{0}^{*}}(s) \\
= & 3 i G^{2} \mu^{4-d} \int \frac{d^{d} l}{(2 \pi)^{d}} \operatorname{Tr}\left[\gamma_{5} G_{q}(l+k) \gamma_{5} G_{Q}(l)\right] \\
= & \frac{3 G^{2}}{8 \pi^{2}}\left\{\frac{4 m_{c}^{2}-2 s}{4-d}+3 m_{c}^{2}-2 s+\left(s-2 m_{c}^{2}\right)\right. \\
& \left.\times\left[\gamma+\ln \left(\frac{m_{c}^{2}-s}{4 \pi \mu^{2}}\right)\right]+\frac{m_{c}^{4}}{s} \ln \left(\frac{m_{c}^{2}-s}{m_{c}^{2}}\right)\right\}
\end{aligned}
$$

$\left(s=k^{2}\right)$. The first term contains the quadratic divergence for $d \rightarrow 4$, showing that the self-energy can be rendered finite with a field and mass renormalization; $\mu$ denotes the mass scale in the dimensional-regularization scheme to keep the momentum dimensions of the integrals as for $d=4$; and $\gamma \simeq 0.577$ is Euler's constant. The (regularization-independent) imaginary part is given by

$$
\operatorname{Im} \Pi_{D}(s)=-\frac{3 G_{S}^{2}}{8 \pi} \frac{\left(s-m_{c}^{2}\right)^{2}}{s} \Theta\left(s-m_{c}^{2}\right) .
$$

For the vector and axial-vector mesons, we employ the HQET propagator for the charm quark,

$$
G_{v}(l)=\frac{m_{c}}{m_{c} v \cdot l+\mathrm{i} \eta} \frac{1+\psi}{2},
$$

to obtain a transverse self-energy in leading order of the expansion in $1 / m_{c}$. In Eq. (11), the "residual momentum" $l$ of the charm quark is defined via its total four-momentum as $l_{c}=m_{c} v+l$. For the $D$-meson fields the corresponding decomposition for the heavy-quark expansion reads

$$
\begin{aligned}
k_{c} & =m_{c} v+k, \quad s=k_{c}^{2}=m_{c}^{2}\left[1+\frac{2 v \cdot k}{m_{c}}+\mathcal{O}\left(\frac{p^{2}}{m_{c}^{2}}\right)\right] \\
& \Rightarrow v \cdot k=\frac{s-m_{c}^{2}}{2 m_{c}}+\mathcal{O}\left(\frac{p^{2}}{m_{c}^{2}}\right) .
\end{aligned}
$$

Applying the Feynman rules, and after some (Dirac) algebra, the dimensionally regularized polarization tensor for the axial vector becomes

$$
\begin{aligned}
\Pi_{D^{*}}^{\mu v}(p)= & \Pi_{D_{1}}^{\mu v}(p)=-6 i G_{V}^{2} \mu^{4-d} m_{c} \\
& \times \int \frac{d^{d} l}{(2 \pi)^{d}} \frac{(l+p)_{v} v_{\mu}-(l+p) \cdot v g_{\mu v}}{\left[(l+p)^{2}+\mathrm{i} \eta\right]\left(m_{c} v \cdot l+\mathrm{i} \eta\right)} .
\end{aligned}
$$

The integral is conveniently evaluated with help of the identity [37]

$$
\frac{1}{a b}=\int_{0}^{\infty} d \lambda \frac{2}{(a+2 \lambda b)^{2}} .
$$

Upon integrating over $\lambda$, one obtains for the imaginary part

$$
\begin{aligned}
\operatorname{Im} \Pi_{D^{*}}^{\mu \nu}(p)= & \operatorname{Im} \Pi_{D_{1}}^{\mu \nu}(p)=-\left(v_{\mu} v_{v}-g_{\mu \nu}\right) \\
& \times \frac{3 G_{V}^{2}}{8 \pi} \frac{\left(s-m_{c}^{2}\right)^{2}}{m_{c}^{2}} \Theta\left(s-m_{c}^{2}\right) .
\end{aligned}
$$

Since up to corrections $\mathcal{O}\left(v k / m_{c}\right)$ one can identify $m_{c}^{2}=s$ in the denominator, one finds

$$
\Pi_{D^{*} \mu \nu}(s)=\Pi_{D_{1} \mu \nu}(s)=\left(v_{\mu} v_{\nu}-g_{\mu \nu}\right) \Pi_{D}(s),
$$

which is the expected result from spin symmetry of the HQET. We define the renormalization constants for the self-energy by the following conditions,

$$
\left.\partial_{s} \Pi_{D}^{(\mathrm{ren})}(s)\right|_{s=0}=0,\left.\quad \operatorname{Re} \Pi_{D}^{\mathrm{ren}}(s)\right|_{s=m_{D}^{2}}=0 .
$$

The first condition ensures that, within a vector dominance model, the photon propagator has residuum of unity at $s=0$, whereas the second one implies that the renormalized meson mass coincides with the bare mass of the Lagrangian. The renormalized self-energy is, of course, independent of the dimensional-regularization scale $\mu$.

As an alternative way of regularizing the divergent self-energy integrals we introduce a dipole form factor at the $c-q-D$ vertex,

$$
F(|\vec{q}|)=\left(\frac{2 \Lambda^{2}}{2 \Lambda^{2}+\vec{q}^{2}}\right)^{2},
$$

where $\vec{q}$ denotes the three-momentum of the quarks in the center-of-mass frame. The imaginary part of the self-energy is then given by

$$
\operatorname{Im} \Pi_{D}^{(\mathrm{ff})}(s)=\operatorname{Im} \Pi_{D}(s) F^{2}(|\vec{q}|),
$$

where $|\vec{q}|=\left(s-m_{c}^{2}\right) /(2 \sqrt{s})$, whereas the real part is determined by an unsubtracted dispersion integral. The bare meson mass is then adjusted to render a vanishing real part of the propagator at the physical resonance mass.

As default parameters for our calculations we use massless light quarks, a charm-quark mass of $m_{c}=1.5 \mathrm{GeV}$, and physical $D$-meson masses $\left(m_{D}^{\text {phys }}\right)^{2}=m_{D}^{2}-\operatorname{Re} \Pi_{D}\left[\left(m_{D}^{\text {phys }}\right)^{2}\right]=$ 
$2 \mathrm{GeV}$ (corresponding to a vanishing real part in the propagator). The coupling constant $G \equiv G_{S, V}$ is varied to allow for widths of the $D$-meson spectral functions of $300-500 \mathrm{MeV}$, to approximately cover the range suggested by effective quark models [32-34]. It is important to note that we assume the $D$-meson resonances to be located above the $c-\bar{q}$ mass threshold, $m_{c}+m_{\bar{q}}$, which renders them accessible in $c-\bar{q}$ scattering processes. The situation is quite different for (bound) meson states (i.e., below the antiquark threshold), where the resonant part of the scattering amplitude cannot be probed through $c+\bar{q} \rightarrow c+\bar{q}$ interactions. (Even for resonance masses close to threshold, thermal energies of antiquarks imply that the average collision energy is significantly above the resonance peak.) In this case, other processes need to be calculated, for example, $c+\bar{q} \rightarrow D+g$, where the extra gluon in the final state carries away four-momentum to allow the $D$ meson to emerge on-shell. The same framework is also applied to the bottom sector, with $b$-quark and $B$-meson masses of $m_{b}=$ $4.5 \mathrm{GeV}$ and $m_{B}=5 \mathrm{GeV}$, respectively.

As we will see in the following, for equal on-shell masses and widths of the $D$-meson spectral functions, both regularization schemes lead to quite comparable results for the thermal relaxation properties of $c$ quarks, even though the off-shell properties differ significantly.

The $D$-meson propagators figure into the invariant matrix elements for elastic $c$-quark scattering off quarks, $c+q \rightarrow$ $c+q$, and antiquarks, $c+\bar{q} \rightarrow c+\bar{q}$, in the $u$ and $s$ channel, respectively. One finds

$$
\begin{aligned}
& \sum\left|\mathcal{M}_{\bar{q}}\right|^{2}=720 G^{4}\left(s-m_{c}^{2}\right)^{2}\left|D_{D}(s)\right|^{2}, \\
& \sum\left|\mathcal{M}_{q}\right|^{2}=720 G^{4}\left(u-m_{c}^{2}\right)^{2}\left|D_{D}(u)\right|^{2},
\end{aligned}
$$

where we have summed over the contributions of all light-quark resonances, which, by heavy-quark symmetry $\left(D-D^{*}, D_{0}^{*}-D_{1}\right)$ or chiral symmetry $\left(D-D_{0}^{*}, D^{*}-D_{1}\right)$, are equal. We also included finite-mass strange quarks along with $D_{s}$ and $D_{s}^{*}$ mesons.

In addition to resonant interactions, elastic scattering in pQCD is accounted for to leading order in $\alpha_{s}, \mathcal{O}\left(\alpha_{s}^{2}\right)$. The corresponding matrix elements [25] have been supplemented with an additional gluon Debye (screening) mass, $\mu_{g}=g T$, which regulates the forward singularity in the $t$-channel exchange graphs [24]. The strong coupling constant will be varied over the range $\alpha_{s}=0.3-0.5$.

\section{CHARM-QUARK RESCATTERING IN THE QGP}

\section{A. Fokker-Planck equation and drag and diffusion coefficients}

The aforementioned matrix elements are now implemented within a kinetic theory framework to assess the thermalization time scales for heavy quarks in a QGP. Following the steps outlined in Ref. [24], we start from the Boltzmann equation for the heavy-quark distribution function $f(t, \vec{p})$ and neglect any mean-field terms. Furthermore, assuming the scattering processes to be dominated by small momentum transfers, one arrives at a Fokker-Planck equation describing the time evolution of $f$ in momentum space,

$$
\frac{\partial f(t, \vec{p})}{\partial t}=\frac{\partial}{\partial p_{i}}\left[A_{i}(\vec{p})+\frac{\partial}{\partial p_{j}} B_{i j}(\vec{p})\right] f(t, \vec{p}) .
$$

For an isotropic (rotationally invariant) plasma, the drag and diffusion coefficients in (22) can be decomposed as

$$
\begin{gathered}
A_{i}(\vec{p})=p_{i} A(|\vec{p}|), \\
B_{i j}(\vec{p})=\left(\delta_{i j}-\frac{p_{i} p_{j}}{\vec{p}^{2}}\right) B_{0}(|\vec{p}|)+\frac{p_{i} p_{j}}{\vec{p}^{2}} B_{1}(|\vec{p}|),
\end{gathered}
$$

with the scalar functions

$$
\begin{gathered}
A(|\vec{p}|)=\langle 1\rangle-\frac{\left\langle\vec{p} \cdot \vec{p}^{\prime}\right\rangle}{\vec{p}^{2}}, \\
B_{0}(|\vec{p}|)=\frac{1}{4}\left[\left\langle\vec{p}^{\prime 2}\right\rangle-\frac{\left\langle\left(\vec{p} \cdot \vec{p}^{\prime}\right)^{2}\right\rangle}{\vec{p}^{2}}\right], \\
B_{1}(|\vec{p}|)=\frac{1}{2}\left[\frac{\left\langle\left(\vec{p} \cdot \vec{p}^{\prime}\right)^{2}\right\rangle}{\vec{p}^{2}}-2\left\langle\vec{p}^{\prime} \cdot \vec{p}\right\rangle+\vec{p}^{2}\langle 1\rangle\right] .
\end{gathered}
$$

The averaging is defined by

$$
\begin{aligned}
\left\langle X\left(\vec{p}^{\prime}\right)\right\rangle= & \frac{1}{2 E_{p}} \int \frac{d^{3} \vec{q}}{(2 \pi)^{3} 2 E_{q}} \int \frac{d^{3} \vec{q}^{\prime}}{(2 \pi)^{3} 2 E_{q^{\prime}}} \int \frac{d^{3} \vec{p}^{\prime}}{(2 \pi)^{3} 2 E_{p^{\prime}}} \frac{1}{\gamma_{c}} \\
& \times \sum|\mathcal{M}|^{2}(2 \pi)^{4} \delta^{(4)}\left(p+q-p^{\prime}-q^{\prime}\right) \hat{f}(\vec{q}) X\left(\vec{p}^{\prime}\right),
\end{aligned}
$$

where $\vec{p}\left(\vec{p}^{\prime}\right)$ and $\vec{q}\left(\vec{q}^{\prime}\right)$ denote the momenta of the incoming (outgoing) charm and light-quark or gluon, respectively, and $\hat{f}(\vec{q})$ are the thermal Maxwell-Boltzmann distribution functions of the light partons. ${ }^{2}$ After integrating over the four-momentum-conserving $\delta$ function, and making use of Lorentz invariance of the matrix elements, we can reduce the expressions for the scalar coefficients, Eqs. (25)-(27), to numerically tractable three-dimensional integrals. ${ }^{3}$

The temperature and momentum dependencies of the $A$ and $B_{0}$ coefficients are summarized in Fig. 2. The main finding

\footnotetext{
${ }^{2}$ As pointed out in Ref. [27] the use of quantum distribution functions (Bose-Einstein and Fermi-Dirac) induces moderate corrections to the drag and diffusion coefficients obtained from $\mathrm{pQCD}$ rescattering; this is still true for the " $D$ "-meson resonance interactions as they involve contributions from thermal antiquarks only, which, at the temperatures under consideration, are mostly nondegenerate. For example, at $T=300 \mathrm{MeV}$, the deviations when using Fermi distributions amount to $\sim 20 \%$ uncertainties associated with the resonance parameters, as discussed in the following. For the total coefficients (including both pQCD and resonance rescattering) the effects of quantum statistics approximately cancel. In the following, we will use Maxwell-Boltzmann distributions for light partons, since (i) it is more consistent within the Fokker-Planck treatment for the heavy quark, the thermal limit of which is a Boltzmann distribution, and (ii) it minimizes the deviations in the dissipation-fluctuation theorem [cf. the discussion following Eq. (45)].

${ }^{3}$ We note that, when using the pQCD matrix elements, our final expressions are larger by a factor of 2 than the ones originally derived in Ref. [24], which confirms the findings of Ref. [27].
} 

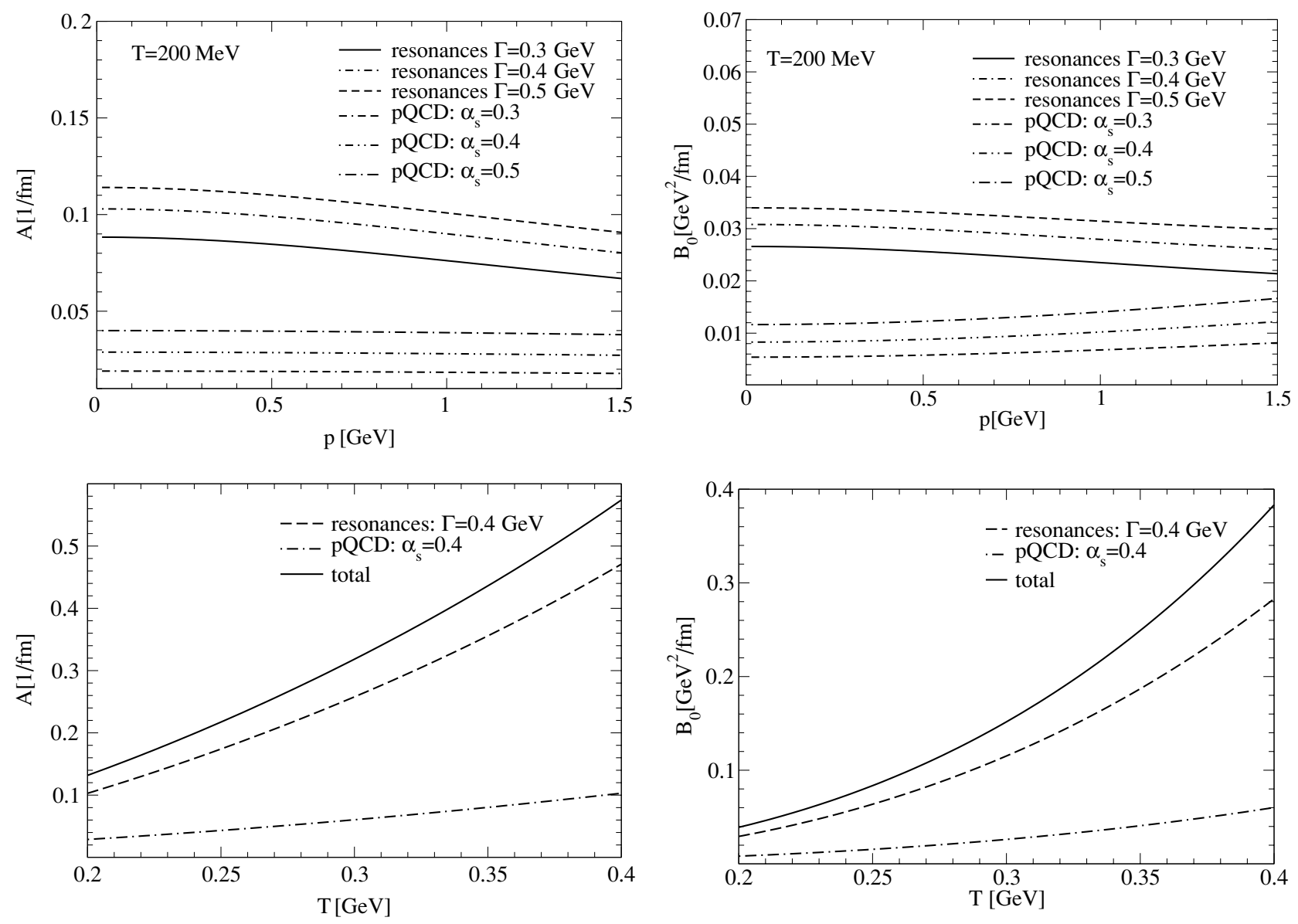

FIG. 2. (Upper panel) Drag coefficient $A$ (left) and diffusion coefficient $B_{0}$ (right) as a function of $c$-quark three-momentum at a temperature of $T=200 \mathrm{MeV}$ for various values of $\alpha_{s}$ (pQCD scattering) and $D$-meson widths (resonance exchanges). (Lower panel) The same quantities as a function of temperature at fixed three-momentum $|\vec{p}|=0$.

here is that for resonant rescattering both are increased by a substantial factor $(\sim 3)$ over the pQCD results. (The latter are very similar to the results of Ref. [24], as the extra factor of 2 is essentially compensated by the larger screening mass, $\mu_{g}=g T$, used in our calculation.) The principal reason for this effect is not so much an increase in the total cross section (the peak cross section in the resonance case of about $10 \mathrm{mb}$ being comparable to an almost constant $4 \mathrm{mb}$ for pQCD) but rather the isotropic angular distribution of the resonance cross sections in contrast to forward dominated pQCD rescattering. Also note that the coefficients are rather insensitive to the underlying coupling constants for both resonance and pQCD scattering; naively, the matrix elements are proportional to the fourth power of the coupling constant, which would imply a variation by a factor of $(5 / 3)^{2} \simeq 2.6$ for the coefficients $A$ and $B_{0}$ for the parameter ranges shown in Fig. 2. The much smaller actual variation is due to compensating effects induced by an increased Debye mass for pQCD $t$-channel gluon exchange, and by an increased resonance width for $s$-channel $D$-meson exchange.

To further illustrate the uncertainties inherent to the resonance properties, we display in Fig. 3 the sensitivity of the drag coefficient with respect to the regularization schemes and the resonance masses (for form-factor regularization). From the left panel one observes that the momentum dependence of the $A$ coefficient is somewhat more pronounced for the form-factor regularization, but the absolute magnitude in both schemes is very similar. The right panel indicates a more pronounced sensitivity to the value of the resonance mass. In particular, it confirms that $D$-meson masses close to threshold lead to a significant reduction of the drag effect, owing to the thermal motion of the light partons from the heat bath. For the same reason, larger masses imply a steeper increase of $A$ with temperature. (Of course, above $T \simeq 2 T_{c}$ the very existence of resonance correlations is questionable.)

\section{B. Time evolution of momentum spectra}

\section{Time dependence of the Fokker-Planck equation}

To obtain a better estimate of the effect of heavy-quark rescattering on the transverse-momentum $\left(p_{T}\right)$ spectra of heavy-flavor hadrons in URHICs we investigate in this section the time evolution of the Fokker-Planck equation in a thermally evolving QGP. The latter is modeled by an expanding fireball under conditions resembling central $\mathrm{Au}-\mathrm{Au}$ 

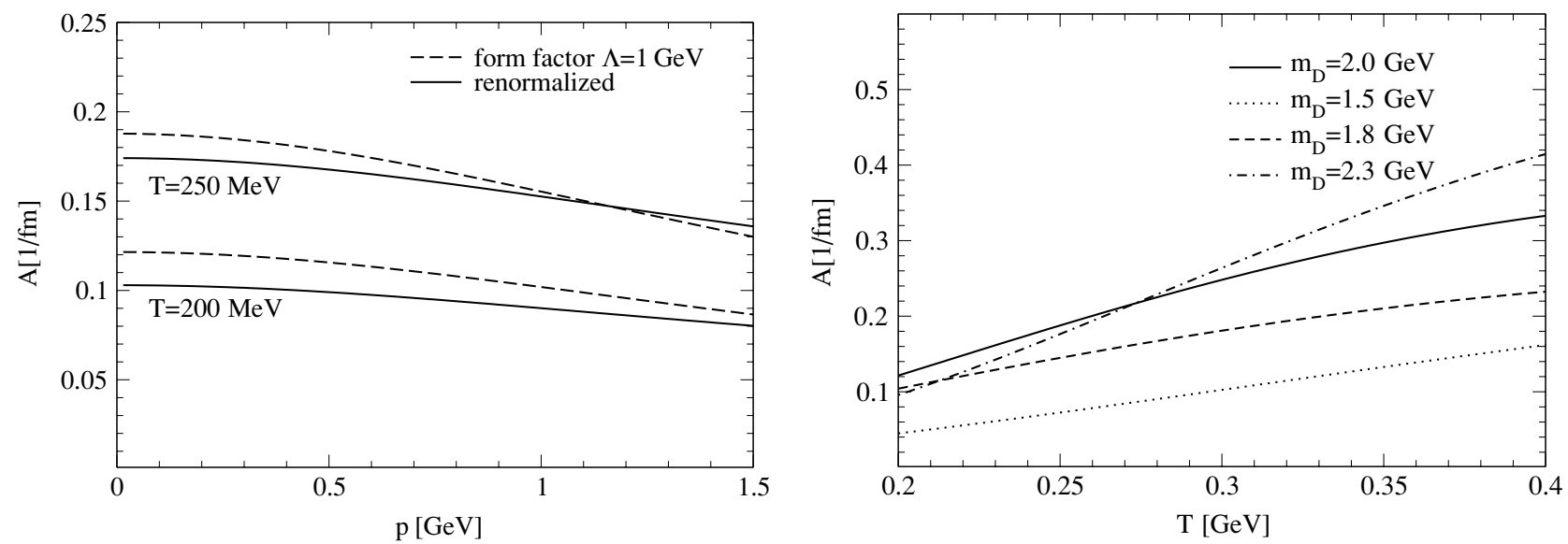

FIG. 3. (Left panel) Comparison of the friction coefficient for resonances with self-energies calculated in the renormalization scheme (17) (solid line) and with form-factor regularization (dashed line). The coupling was chosen such that, in both, the resonance width $\Gamma=0.4 \mathrm{GeV}$ at the resonance mass. (Right panel) The friction coefficient for different resonance masses as function of the temperature, evaluated in the form-factor regularization scheme.

collisions at RHIC. The main simplifying assumption consists of momentum-independent drag and diffusion coefficients. According to the discussion at the end of the previous section, we will therefore employ $D$-meson self-energies within the renormalization scheme (17) (with masses and widths of $m_{D}=2 \mathrm{GeV}$ and $\Gamma=0.4 \mathrm{GeV}$ ), as well as pQCD cross sections (with $\alpha_{s}=0.4$ ) (cf. Fig. 2). The Fokker-Planck equation (22) then takes the form

$$
\frac{\partial f}{\partial t}=\gamma(t) \frac{\partial}{\partial \vec{p}}(\vec{p} f)+D(t) \frac{\partial^{2}}{\partial \vec{p}^{2}} f,
$$

where $\gamma=A(T(t),|\vec{p}|=0)$ and $D=B_{0}(T(t),|\vec{p}|=0)=$ $B_{1}(T(t),|\vec{p}|=0)$. The time dependence of the coefficients enters through their dependence on temperature (as determined in the previous section), with $T(t)$ following from the fireball model outlined in the following. For the initial condition,

$$
f(t=0, \vec{p}) \equiv f_{0}(\vec{p}),
$$

we will employ $c$-quark spectra from $p$ - $p$ collisions as extracted from the PYTHIA event generator [38,39]. The initial-value problem can be conveniently solved employing Green's function techniques: If we can find a solution $G\left(t, \vec{p} ; \vec{p}_{0}\right)$ to Eq. (29) with the initial condition

$$
G\left(t=0, \vec{p} ; \vec{p}_{0}\right)=\delta^{(3)}\left(\vec{p}-\vec{p}_{0}\right),
$$

the full solution with an arbitrary initial condition (30) follows as

$$
f(t, \vec{p})=\int d^{3} \vec{p}_{0} G\left(t, \vec{p} ; \vec{p}_{0}\right) f_{0}\left(\vec{p}_{0}\right) .
$$

To determine the Green's function, we define its Fourier transform,

$$
G\left(t, \vec{p} ; \vec{p}_{0}\right)=\int d^{3} \vec{q} \exp (-i \vec{q} \cdot \vec{p}) g\left(t, \vec{q} ; \vec{p}_{0}\right),
$$

and insert it into Eq. (29), leading to the first-order differential equation

$$
\frac{\partial g}{\partial t}+\gamma \vec{q} \frac{\partial g}{\partial \vec{q}}=-D \vec{q}^{2} g .
$$

With the initial condition for $g$ determined by Eq. (31),

$$
g\left(0, \vec{q}, \vec{p}_{0}\right)=\frac{1}{(2 \pi)^{3}} \exp \left(-i \vec{p}_{0} \cdot \vec{q}\right),
$$

its solution reads

$$
g\left(t, \vec{q} ; \vec{p}_{0}\right)=\frac{1}{(2 \pi)^{3}} \exp \left\{i \vec{p}_{0} \cdot \vec{q} \exp [-\Gamma(t)]\right\} \exp \left[-\Delta(t) \vec{q}^{2}\right],
$$

where

$$
\Gamma(t)=\int_{0}^{t} d \tau \gamma(\tau)
$$

and

$$
\Delta(t)=\exp [-2 \Gamma(t)] \int_{0}^{t} d \tau D(\tau) \exp [2 \Gamma(\tau)] .
$$

The Fourier transformation (33) yields the result for the Green's function,

$$
G\left(t, \vec{p} ; \vec{p}_{0}\right)=\left[\frac{1}{4 \pi \Delta(t)}\right]^{3 / 2} \exp \left\{-\frac{\left(\vec{p}-\vec{p}_{0} \exp [-\Gamma(t)]\right)^{2}}{4 \Delta(t)}\right\},
$$

and the time evolution of the distribution function, Eq. (32), is finally obtained by numerical integration.

\section{Limiting cases}

Before we turn to the full solution, let us first illustrate a few limiting cases. For time-independent coefficients $\gamma$ and $D$, Eqs. (37) and (38) simplify to

$$
\Gamma(t)=\gamma t, \quad \Delta(t)=\frac{D}{2 \gamma}[1-\exp (-2 \gamma t)] .
$$



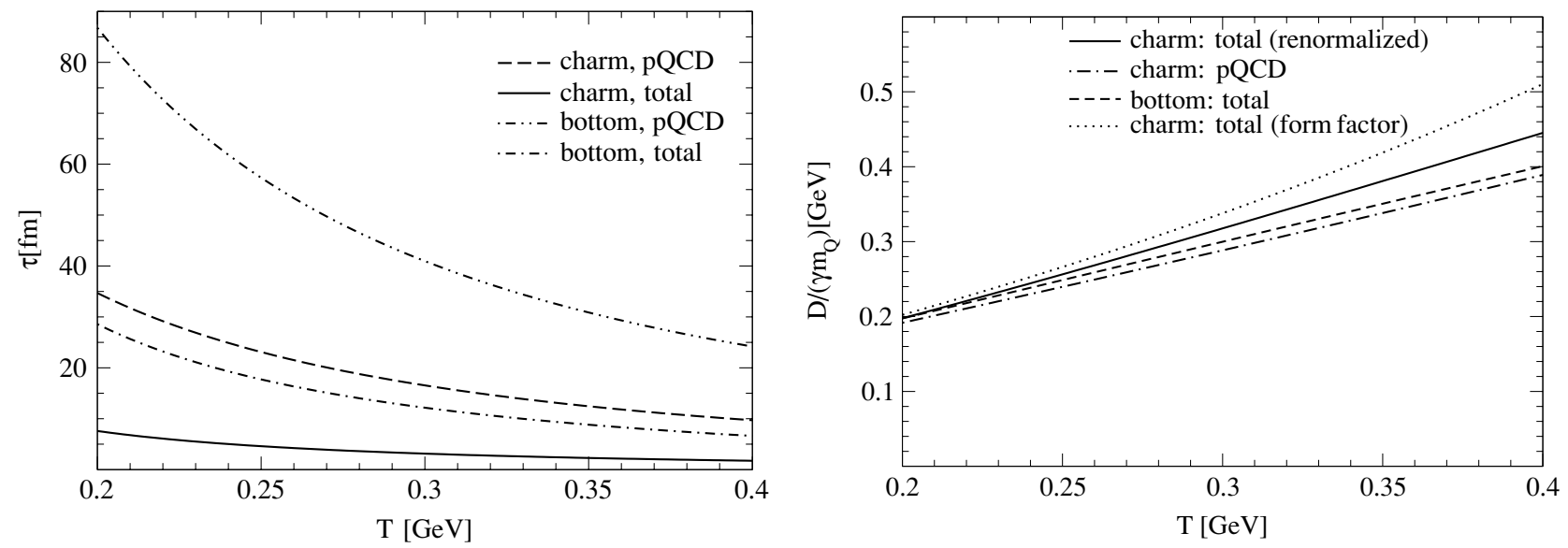

FIG. 4. (Left panel) Equilibration time scale $\tau=1 / \gamma$ for charm and bottom quarks in the QGP as function of temperature with (solid and dashed lines) and without (dash-dotted and dash-double-dotted lines) in-medium resonances. (Right panel) Consistency check of the dissipation-fluctuation relation, Eq. (45), for $c$ quarks with (solid line) and without (dash-dotted line) resonances, as well as for $b$ quarks (with resonances, dashed line), in the QGP.

Inserting these into Eq. (39) yields

$$
\begin{aligned}
G\left(t, \vec{p} ; \vec{p}_{0}\right)= & \left\{\frac{\gamma}{2 \pi D[1-\exp (-2 \gamma t)]}\right\}^{3 / 2} \\
& \times \exp \left\{-\frac{\gamma}{2 D} \frac{\left[\vec{p}-\vec{p}_{0} \exp (-\gamma t)\right]^{2}}{1-\exp (-2 \gamma t)}\right\},
\end{aligned}
$$

which was already derived in Ref. [24]. This, in particular, shows that $\gamma$ has the meaning of a drag (or friction) coefficient,

$$
\langle\vec{p}(t)\rangle=\vec{p}_{0} \exp (-\gamma t)
$$

characterizing the equilibration time scale $\tau=1 / \gamma$. With the momentum fluctuation given by

$$
\left\langle\vec{p}^{2}(t)\right\rangle-\langle\vec{p}(t)\rangle^{2}=\frac{3 D}{\gamma}[1-\exp (-2 \gamma t)],
$$

$D$ is readily identified as a momentum diffusion coefficient. From the left panel of Fig. 4 we see that, for temperatures expected in central Au-Au collisions at RHIC, the equilibration time scale for charm quarks is substantially reduced by resonant interactions, to about a few $\mathrm{fm} / c$, comparable to the duration of the (putative) QGP phase. Even though a similar mechanism is operative for bottom quarks, their much larger rest mass renders the pertinent kinetic equilibration time significantly larger, around $10 \mathrm{fm} / c$ or more.

For $t \rightarrow \infty$, Eq. (41) approaches a Maxwell-Boltzmann distribution,

$$
\lim _{t \rightarrow \infty} G\left(t, \vec{p} ; \vec{p}_{0}\right)=f_{\mathrm{eq}}(\vec{p})=\left(\frac{\gamma}{2 \pi D}\right)^{3 / 2} \exp \left[-\frac{\gamma \vec{p}^{2}}{2 D}\right],
$$

and thermal equilibrium implies the dissipation-fluctuation theorem,

$$
T=\frac{D}{\gamma m_{c}} .
$$

Such an obtained temperature $T$ should, of course, coincide with that of the (light-quark and gluon) heat bath entering through the thermal distribution functions in Eq. (28). Thus,
Eq. (45) serves as a consistency check for the determination of the drag and diffusion coefficients within our model, especially for the assumption on the dominance of small momentum transfers underlying the Fokker-Planck equation (22). From the right panel in Fig. 4 we see that, for charm quarks, the dissipation-fluctuation theorem is well satisfied (within $3 \%$ ) when using forward peaked pQCD cross sections; but also for the isotropic resonance cross sections within the renormalization scheme the deviations do not exceed $11 \%$ even at the highest considered temperatures, while within the form-factor regularization scheme they reach up to $26 \%$ (the latter value is reduced to $\sim 17 \%$ when an average over a thermal momentum distribution is performed). The latter is due to the greater variation of the friction and diffusion coefficients with momentum, which makes the approximation of momentumindependent coefficients underlying the derivation of the fluctuation-dissipation relation (45) less accurate. For this reason, in the next section we shall use the renormalization scheme without form factor to investigate the time evolution of $p_{T}$ spectra. For the heavier bottom quarks the fluctuationdissipation theorem is satisfied to high accuracy.

\section{Charm-quark $p_{T}$ spectra at RHIC}

Let us now address the time evolution of charm-quark $p_{T}$ spectra including the temperature dependence of drag and diffusion coefficients. To obtain the time evolution of the temperature we use a simple expanding fireball model [40]. In reminiscence to hydrodynamic simulations [41] of central $\mathrm{Au}-\mathrm{Au}$ collisions at RHIC, the fireball volume is parametrized by

$$
V_{\mathrm{FB}}(t)=\pi\left(z_{0}+v_{z} t\right)\left(r_{0}+\frac{1}{2} a_{\perp} t^{2}\right)^{2},
$$

where $r_{0}=6.5 \mathrm{fm}$ and $z_{0}=0.6 \mathrm{fm}$ are the initial transverse and longitudinal size, respectively (the latter corresponding to a formation time of $\tau_{0}=0.33 \mathrm{fm} / c$ ). The longitudinal and transverse expansions are characterized by $v_{z}=1.4 c$ 

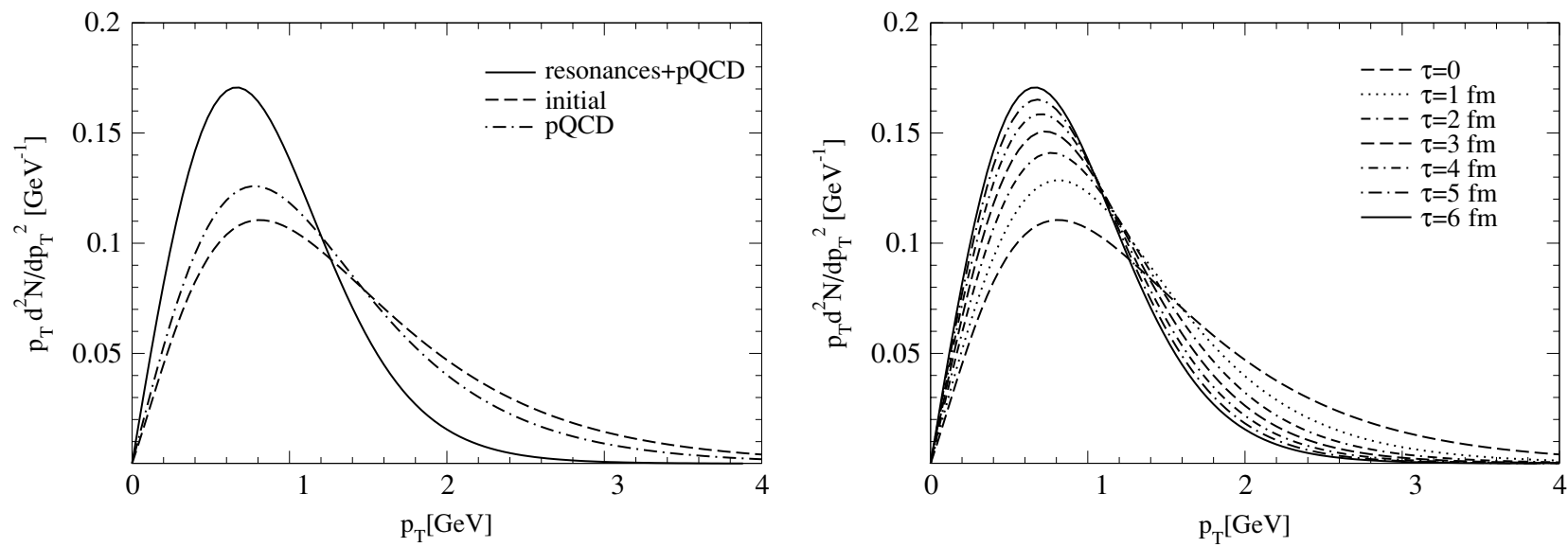

FIG. 5. (Left panel) Results for the time-evolved $c$-quark $p_{T}$ spectra in the local rest frame with a temperature profile corresponding to QGP and mixed phase in central Au-Au collisions at $\sqrt{s_{N N}}=200 \mathrm{GeV}$; dashed curve: initial spectrum taken from $p$ - $p$ collisions; dash-dotted curve: final spectrum using pQCD cross sections only; solid curve: final spectrum using both pQCD and $D$-meson resonance interactions. (Right panel) Explicit time evolution in time steps of $1 \mathrm{fm} / c$ for the pQCD+resonance interactions.

(covering a thermal width of about 1.8 units in rapidity) and $a_{\perp}=0.055 c^{2} / \mathrm{fm}$ (yielding a total fireball lifetime of about $14 \mathrm{fm} / c$ with a thermal freezeout temperature of $\sim 110 \mathrm{MeV}$ ).

Assuming isentropic expansion, the temperature at each instant is calculated from the total entropy of produced particles, $S=s(T) V_{\mathrm{FB}}(t) \simeq 10^{4}$ (within $\Delta y=1.8$ ), with the entropy density in the QGP given by

$$
s=\frac{4 \pi^{2}}{90} T^{3}\left(16+10.5 N_{f}\right)
$$

where $N_{f}$ is the effective number of quark flavors, taken to be 2.5 .

With these parameters, the initial QGP temperature is $T_{0} \simeq 375 \mathrm{MeV}$, decreasing to the critical temperature of $T_{c} \simeq 180 \mathrm{MeV}$ after about $3 \mathrm{fm} / c$, with further evolution in a hadron-QGP mixed phase for another $3 \mathrm{fm} / c$. For simplicity, we treat the latter phase as a QGP at constant $T=T_{c} \simeq$ $180 \mathrm{MeV}$, but account for lower parton densities as estimated from the temperature dependence of the Fokker-Planck coefficients. (From the lower panel of Fig. 2 we estimated $A, B \propto \varrho^{2 / 3}$, where $\varrho$ denotes the density of the light partons.)

For the initial distribution $f_{0}(\vec{p})$, Eq. (30), we employ charm-quark $p_{T}$ spectra as generated in proton-proton $(p-p)$ collisions at $200 \mathrm{GeV}$ by PYTHIA [38]. A suitable parametrization thereof is given by [39]

$$
\frac{d^{2} N_{c}}{d p_{T}^{2}}=C \frac{\left(p_{T}+A\right)^{2}}{\left(1+p_{T} / B\right)^{\alpha}}
$$

with $A=0.5 \mathrm{GeV}, \quad B=6.8 \mathrm{GeV}, \quad \alpha=21$, and $C=$ $0.845 \mathrm{GeV}^{-4}$.

As already detailed, the time evolution of the $p_{T}$ distribution is obtained from Eq. (32) with the Green's function (41), integrated over $p_{z}$, which amounts to a two-dimensional solution of the Fokker-Planck equation.

Figure 5 shows the time evolution of $c$-quark $p_{T}$ spectra in central $\mathrm{Au}-\mathrm{Au}$ at RHIC, evaluated in the local (thermal) rest frame of the expanding matter in QGP and mixed phase (i.e., the additional boost from the collective transverse expansion is not included), using the drag and diffusion coefficients computed in Sec. III A. From the left panel one finds that, when allowing for $\mathrm{pQCD}$ rescattering only, the initial spectra from $p-p$ collisions are affected rather little. In contrast, when augmenting the interactions with $D$-meson resonances, ${ }^{4}$ the $p_{T}$ spectra undergo a marked reshaping, essentially a redistribution from high to low $p_{T}$. The final spectra (i.e., at the end of the mixed phase after $6 \mathrm{fm} / c$ ), with a peak position at $p_{T}^{(\max )} \simeq 0.66 \mathrm{GeV}$, indeed closely resemble a thermal distribution with a temperature of about $290 \mathrm{MeV}$. Even though this indicates that the spectra are not fully thermalized at $T_{c}$, the change from an initial average $\sqrt{\left\langle p_{T}^{2}\right\rangle}=$ $1.66 \mathrm{GeV}$ (corresponding to a "temperature" of $\sim 920 \mathrm{MeV}$ ) is appreciable. The fact that most of the rescattering occurs in the early evolution phase (the first $3 \mathrm{fm} / c$ or so; cf. right panel of Fig. 5) should provide favorable conditions for the buildup of elliptic flow.

We recall that our treatment becomes unreliable toward high $p_{T}$, since (i) we have neglected the momentum dependence of the drag and diffusion coefficients (i.e., used their values at zero momentum), whereas in reality they decrease with $p_{T}$ (cf. Fig. 2), and (ii) we have not accounted for induced gluon emission, which is expected to be the main mechanism for energy loss of high- $p_{T}$ partons within pQCD $[5,6]$. Also note that transverse-flow effects, that is, Lorentz boosts of the (partially) thermalized $c$ quarks from the comoving (thermal) frame into the lab frame, are not included in Fig. 5. Finally, the (possibly gradual) disappearance of resonance states toward high temperatures has not been incorporated. (Whether this happens under RHIC conditions with initial temperatures of

\footnotetext{
${ }^{4}$ Note that, when adding the matrix elements for $\mathrm{pQCD}$ and resonance interactions, we did not resum the perturbative contributions to, say, $c+\bar{q} \rightarrow c+\bar{q}$ in the $D$-meson resonance propagator. In principle, this would lead to a slight renormalization of the resonance mass and width, which, however, is well inside the range of uncertainties of the in-medium resonance parameters.
} 
$2 T_{c}$ is not clear at present; e.g., in the lattice calculations of Refs. [30,31] resonance signals are still observed around these temperatures.)

\section{CONCLUSIONS AND OUTLOOK}

In the present work we have studied the role of resonant rescattering for heavy quarks in a quark-gluon plasma at moderate temperatures. Our main assumption has been the existence of $D$-meson-like resonance states above $T_{c}$, which finds support in both effective quark models and recent QCD lattice calculations. The underlying Lagrangian embodied both chiral and heavy-quark symmetry, where the latter has been essential to ensure conserved vector currents (for $D^{*}$ resonances). Bare masses and coupling constants of the model have been adjusted to render one-loop resummed $D$-meson spectral functions reminiscent of more microscopic calculations.

Pertinent cross sections for resonant $c$-quark rescattering on light antiquarks in the heat bath have been applied within a Fokker-Planck equation to evaluate kinetic thermalization. Our main finding is that the introduction of resonances in the QGP leads to a substantial reduction of the equilibration-time scales (by a factor of $\sim 3$ ) compared to estimates based on perturbative interactions. To a large extent, this difference originates from the isotropy of the angular distributions for resonant scattering, as opposed to mostly forward scattering in pQCD, which importantly enters into the (angular-weighted) transport cross section. The reduced time scales are very reminiscent of the expected QGP lifetimes in central Au-Au collisions at RHIC. Consequently, time-evolved $c$-quark $p_{T}$ spectra exhibit a marked tendency toward thermalization in the resonance picture, whereas they are only little affected by pQCD rescattering alone. This should have important consequences for the buildup of elliptic flow of charmed hadrons.

Let us briefly discuss further ramifications and directions for future work. Clearly, the three-momentum dependence of the drag and diffusion coefficients, the effects of transverse flow, and the evaluation of elliptic flow need to be addressed. Quasiparticle masses for light quarks and gluons should be introduced to make a closer connection to the QGP equation of state. The disappearance of the bound states needs to be accounted for, especially for applications at the Large Hadron Collider (LHC) with larger anticipated initial temperatures than at RHIC. Indeed, a key question that may eventually be answered by lattice QCD is whether $D$-meson (and other) resonances in the QGP exist, and, if so, whether they are located above the two-quark threshold, which renders them accessible for direct $(c+\bar{q} \rightarrow c+\bar{q})$ scattering processes. Clearly, if this is not the case, other processes, such as $c+$ $\bar{q} \rightarrow D+g$, need to be evaluated. The effects on secondary $c \bar{c}$ production should also be checked [42]. For example, according to recent transport calculations [29], upscaling pQCD cross sections by a factor of 3 (to generate a significant elliptic flow) entails an increase in open-charm pairs by $40-50$ at RHIC, which is not supported by current PHENIX data [43]. Resonance cross sections, owing to the larger $D$-meson mass in the crossed channel, may not have this feature (or, at least, have it to a lesser extent). Finally, we recall the important impact that heavy-quark momentum distributions have on secondary production ("coalescence") of charmonium and bottomonium states. Obviously, the combined theoretical and experimental study of heavy-flavor probes promises a rich potential for understanding the complex nature of QGP at moderate temperatures.

\section{ACKNOWLEDGMENTS}

One of us $(\mathrm{HvH})$ thanks the Feodor Lynen program of the Alexander von Humboldt foundation for financial support. This work was supported in part by a U.S. National Science Foundation CAREER award under Grant PHY-0449489.
[1] R. Vogt, Phys. Rep. 310, 197 (1999).

[2] H. Satz, Rep. Prog. Phys. 63, 1511 (2000).

[3] R. Rapp and L. Grandchamp, J. Phys. G 30, S305 (2004).

[4] D. Kharzeev and K. Tuchin, Nucl. Phys. A735, 248 (2004).

[5] M. Djordjevic, M. Gyulassy, and S. Wicks, hep-ph/0410372.

[6] N. Armesto, C. A. Salgado, and U. A. Wiedemann, Phys. Rev. D 69, 114003 (2004).

[7] E. L. Bratkovskaya, W. Cassing, H. Stöcker, and N. Xu, nuclth/0409047.

[8] S. Batsouli, S. Kelly, M. Gyulassy, and J. L. Nagle, Phys. Lett. B557, 26 (2003).

[9] V. Greco, C. M. Ko, and R. Rapp, Phys. Lett. B595, 202 (2004).

[10] S. Kelly et al. (PHENIX Collaboration), J. Phys. G 30, S1189 (2004).

[11] F. Laue et al. (STAR Collaboration), nucl-ex/0411007.

[12] E. V. Shuryak and I. Zahed, Phys. Rev. C 70, 021901(R) (2004).

[13] G. E. Brown, C.-H. Lee, M. Rho, and E. Shuryak, Nucl. Phys. A740, 171 (2004).

[14] X. Li, H. Li, C. M. Shakin, and Q. Sun, Phys. Rev. C 69, 065201 (2004).
[15] S. Datta, F. Karsch, P. Petreczky, and I. Wetzorke, Nucl. Phys. Proc. Suppl. 119, 487 (2003).

[16] T. Umeda, K. Nomura, and H. Matsufuru, hep-lat/0211003.

[17] L. Grandchamp, R. Rapp, and G. E. Brown, Phys. Rev. Lett. 92, 212301 (2004).

[18] R. L. Thews, M. Schroedter, and J. Rafelski, Phys. Rev. C 63, 054905 (2001).

[19] B. Zhang, C. M. Ko, B.-A. Li, Z.-W. Lin, and S. Pal, Phys. Rev. C 65, 054909 (2002).

[20] P. Braun-Munzinger and J. Stachel, Phys. Lett. B490, 196 (2000).

[21] M. I. Gorenstein, A. P. Kostyuk, H. Stöcker, and W. Greiner, Phys. Lett. B509, 277 (2001).

[22] R. L. Thews, hep-ph/0206179.

[23] L. Grandchamp and R. Rapp, Nucl. Phys. A709, 415 (2002).

[24] B. Svetitsky, Phys. Rev. D 37, 2484 (1988).

[25] B. L. Combridge, Nucl. Phys. B151, 429 (1979).

[26] M. G. Mustafa and M. H. Thoma, hep-ph/0311168.

[27] M. G. Mustafa, D. Pal, and D. K. Srivastava, Phys. Rev. C 57, 889 (1998). 
[28] L.-W. Chen and C. M. Ko, nucl-th/0409058.

[29] D. Molnar, nucl-th/0410041.

[30] M. Asakawa and T. Hatsuda, Nucl. Phys. A721, 869 (2003).

[31] F. Karsch and E. Laermann (2003), hep-lat/0305025, in QuarkGluon Plasma III, edited by R. C. Hwa and X.-N. Wang (World Scientific, Singapore, 2004), p. 1.

[32] F. O. Gottfried and S. P. Klevansky, Phys. Lett. B286, 221 (1992).

[33] D. Blaschke, G. Burau, T. Barnes, Y. Kalinovsky, and E. Swanson, Heavy Ion Phys. 18, 49 (2003).

[34] D. Blaschke, G. Burau, Y. L. Kalinovsky, and V. L. Yudichev, Prog. Theor. Phys. Suppl. 149, 182 (2003).

[35] D. Ebert, T. Feldmann, R. Friedrich, and H. Reinhardt, Nucl. Phys. B434, 619 (1995).
[36] P. Ramond, Field Theory: A Modern Primer (Addison-Wesley, Redwood City, CA,1989), 2nd ed.

[37] H. Georgi, Boulder TASI 91 589-630 (1991).

[38] T. Sjostrand, L. Lonnblad, S. Mrenna et al., Comput. Phys. Commun. 135, 238 (2001).

[39] V. Greco (private communication).

[40] R. Rapp, Phys. Rev. C 63, 054907 (2001).

[41] P. F. Kolb and R. Rapp, Phys. Rev. C 67, 044903 (2003).

[42] P. Levai, B. Müller, and X.-N. Wang, Phys. Rev. C 51, 3326 (1995).

[43] S. S. Adler et al. (PHENIX Collaboration), nucl-ex/ 0409028. 